\title{
Gait performance of the elderly under dual-task conditions: Review of instruments employed and kinematic parameters
}

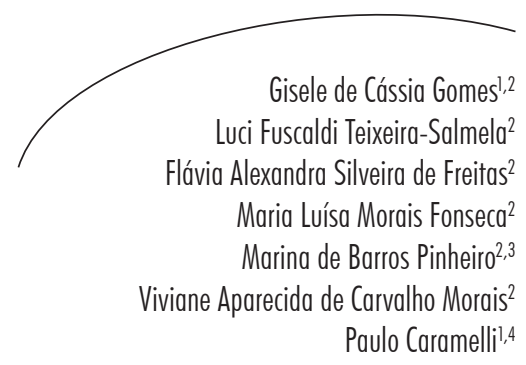

Abstract

Introduction: The physiological deterioration associated with ageing exposes elderly persons to greater risks of falls, especially during the performance of simultaneous tasks during gait. Objectives: To evaluate the effects of dual tasks (DT) on spatiotemporal gait parameters and to identify the tools and tasks most commonly used to assess the performance of DT among the elderly. Method: Searches of the MEDLINE, PsycINFO, CINAHL, and SciELO databases were conducted. Observational studies, which evaluated gait changes during the performance of DT, published up to April 2014, were selected. Results: A total of 385 articles were found, of which 28 were selected. Decreases in speed and increases in stride variability, stride time, step width, and double support time were observed under DT conditions. Motion analysis systems, such as the GAITRite walkway ${ }^{\circledR}$ system were the mostly commonly used instruments for the analyses of kinematic parameters (16 studies). DT was most commonly assessed by arithmetic calculations in 20 studies, followed by verbal fluency, in nine studies. The gait parameters most commonly assessed were speed (19 studies), followed by stride variability (14 studies). Conclusion: The elderly showed changes in spatiotemporal gait parameters under DT conditions. Gait speed and stride variability were often assessed and, together, were considered good indicators of risks of falls.
Keywords: Aging; Elderly; Gait; Geriatric Assessment; Elderly Health.

Universidade Federal de Minas Gerais, Programa de Pós-graduação em Neurociências. Belo Horizonte, MG, Brasil.

2 Universidade Federal de Minas Gerais, Escola de Educação Física, Fisioterapia e Terapia Ocupacional, Departamento de Fisioterapia. Belo Horizonte, MG, Brasil.

3 The University of Sydney, Faculty of Health Sciences. Arthritis \& Musculoskeletal Research Group AMRG. Cumberland Campus. Sydney, Austrália.

4 Universidade Federal de Minas Gerais, Faculdade de Medicina, Departamento de Clínica Médica. Belo Horizonte, MG, Brasil.

Correspondence

Gisele de Cássia Gomes

Departamento de Fisioterapia, Universidade Federal de Minas Gerais

Av. Antônio Carlos, 6627, Campus Pampulha

CEP: 31270-901, Belo Horizonte, MG, Brazil

E-mail: giselecg@ufmg.br 


\section{INTRODUCTION}

Life expectancy and the proportion of elderly individuals in the population are increasing worldwide. ${ }^{1}$ This has led to reflection on a number of issues, including quality of life, physical and mental wellbeing ${ }^{1}$ and the possibility of disabling events that reduce the functional capacity of this population. ${ }^{2}$ As an individual gets older, they experience a cumulative physiological decline in different bodily systems, which is characterized by structural and functional abnormalities. ${ }^{3}$ These abnormalities involve alterations that compromise the performance of motor skills, including impaired postural control, abnormal posture/gait/balance, a reduction in functional capacity and difficulties in adapting to the environment, all of which can lead to a greater risk of falls., ${ }^{2,3}$

Falls generally occur while an individual is walking. ${ }^{1,4}$ For elderly individuals, walking demands a greater quantity of attentional resources and different cognitive mechanisms to maintain adequate control and performance levels. ${ }^{4}$ Elderly individuals have shown an inability to adapt their walking pattern to unexpected situations in their daily lives, which usually occur when performing several simultaneous tasks. ${ }^{4-6}$

The dual-task (DT) paradigm has been in use since the middle of the 1980s and involves the performance of two concomitant tasks. It was first used in psychological studies and was later adopted by health professionals working in the areas of rehabilitation and gerontology to assess and train elderly individuals in a secondary task, with walking as the primary task. ${ }^{7}$ This paradigm seeks to assess the effects of cognitive and motor resources on the stability of an individual's gait, postural control and falls. In addition, the DT paradigm favors the detection of gait issues, as well as possible cognitive deficits, which may go undetected when assessed using a single task (ST). ${ }^{4}$

A poor performance in a walking related DT has been strongly correlated with falls, particularly among the elderly population, given that these individuals require greater motor control to maintain their balance. This is due to the fact that postural control, motor tasks (MT) and cognitive tasks (CT) are processed at a cortical level among the elderly, allowing one activity to interfere with the other, leading to a deviation or a reduction in the attentional resources for one of the tasks. ${ }^{8,9}$ When the performance of one task interferes with the performance of another, a negative interaction can occur between the tasks, which leads to the individual exceeding the capacity of the resource available, even when the tasks have been previously learned or are performed automatically., ${ }^{40,11}$ Thus, the danger of executing one of the tasks will be proportional to the amount of care that the other task demands. ${ }^{12}$ Another relevant factor is that elderly individuals have greater difficulty in performing a DT than younger individuals. ${ }^{13}$

Walking is a complex motor task, with limited involvement of cognitive control, and its performance is considered automatic in healthy adults, although this automatism is lost in old age., ${ }^{4,14}$ The aging process is accompanied by alterations in the spatial and temporal parameters of gait, ${ }^{14}$ such as reductions in gait speed and step length, as well as increases in step variability, double support time and step width, ${ }^{4,12,15}$ all of which have been correlated with a higher incidence of falls. ${ }^{5,6,16-21} \mathrm{An}$ assessment of these parameters is generally used to identify abnormalities in an individual's gait. ${ }^{22}$

While several gait parameters (reduced speed, reduced step length and increased double support time) can reflect impairment and are correlated with a fear of falls, step variability is the indicator that provides the best representation of postural instability. ${ }^{23}$ Of the gait parameters that are usually assessed in studies, variability in the speed of the individual's steps has been shown to be the strongest fall predictor for the elderly. ${ }^{24}$ Greater step variability (greater gait irregularity) in isolation, without the need to perform a simultaneous task, has also been shown to be a strong fall predictor among community-dwelling elderly individuals. ${ }^{16}$ Step time addresses the control and regularity of the rhythm of gait. ${ }^{12}$ 
Considering the difficulty that elderly individuals have in reacting to unexpected situations in their daily lives, such as when they need to perform simultaneous tasks while walking, ${ }^{4-6}$ as well as the correlation between this activity and the occurrence of falls, ${ }^{8,9}$ it is important to study the alterations to gait parameters that occur during the performance of dual-tasks and to determine the adequacy of the instruments used to assess these parameters.

Therefore, the aim of this review was to assess the effects of performing a dual-task on the spatial and temporal parameters of the gait of elderly individuals and to identify which instruments are most commonly used to assess the gait of elderly individuals during the performance of a dual-task. The results of this review could contribute to our understanding of the motor control mechanisms of elderly individuals while performing a DT and help the assessment and treatment of elderly individuals that exhibit abnormal gait/balance and are at risk of falls.

\section{METHODS}

For this review, searches were conducted in the following electronic databases: MEDLINE (Ovid); PsycINFO (Ovid); CINAHL (EBSCO) and SciELO. Specific and optimized search strategies were used for each database, by combining the following descriptors and keywords: aging, aged, elder, elderly, in relation to the elderly; dual-task, multitask, triple-task, in relation to DT and; gait, walk, gait analysis and locomotion, in relation to gait.

The searches were performed up to April of 2014, with no restrictions applied for the initial date of publication. The following search strategy was used for the MEDLINE and PsycINFO databases: (Exp Aging/ OR Elderly.mp OR Exp *aged OR "aged, 80 and over"/ OR Aging.mp OR elder\$. tw) AND (Dual task.mp OR Dual task $\$ . m p$ OR (dual adj2 task).mp OR Dual-task\$.mp OR Multi task\$.tw OR Mukti-task\$.tw OR Triple task\$.tw) AND (Gait/ OR Gait analysis.mp OR Walking/
OR Locomotion/). The search strategy used for the other databases was an adaptation of the MEDLINE search.

The following inclusion criteria were used: (1) study type: observational; (2) population: elderly individuals with no impairment of the central and peripheral nervous systems or bone and musculoskeletal systems; (3) outcome measurements: spatial and temporal parameters of gait in association with a DT, including gait speed, step length and step variability, which are measured using electronic motion analysis systems (MAS), insoles, cameras, chronometers; (4) language: Spanish, French, English and Portuguese. Study selection was carried out by two independent examiners, and, in cases of disagreement, a third examiner was involved in the final decision. Initially, the titles and abstracts were read, in accordance with the established inclusion criteria. After the first exclusion, the selected articles were analyzed in full. Subsequently, a manual search was conducted in the references section of the articles selected.

The following study data was included: a description of the sample; the spatial and temporal parameters of gait that were analyzed; the frequency of the tasks and the instruments used.

\section{RESULTS}

The database searches returned a total of 385 articles, of which 104 were excluded for duplicity. The titles and abstracts of the remaining 281 articles were assessed and a further 246 were excluded for not fulfilling the inclusion criteria. Thus, 35 studies were selected for a full reading. After this process had been completed, seven studies were excluded as they did not assess gait during the performance of a DT as the main outcome $(n=6)$ or they were a review $(\mathrm{n}=1)$. In total, 28 articles that had been published up to April 2014 were included in this review. Figure 1 outlines the study selection process. 
Studies found after searching the databases $(n=385)$

- MEDLINE $(n=118)$

- PsyciNFO $(n=60)$

- CINAHL $(n=103)$

- SciELO $(n=4)$

Studies found after active manual search $(n=0)$

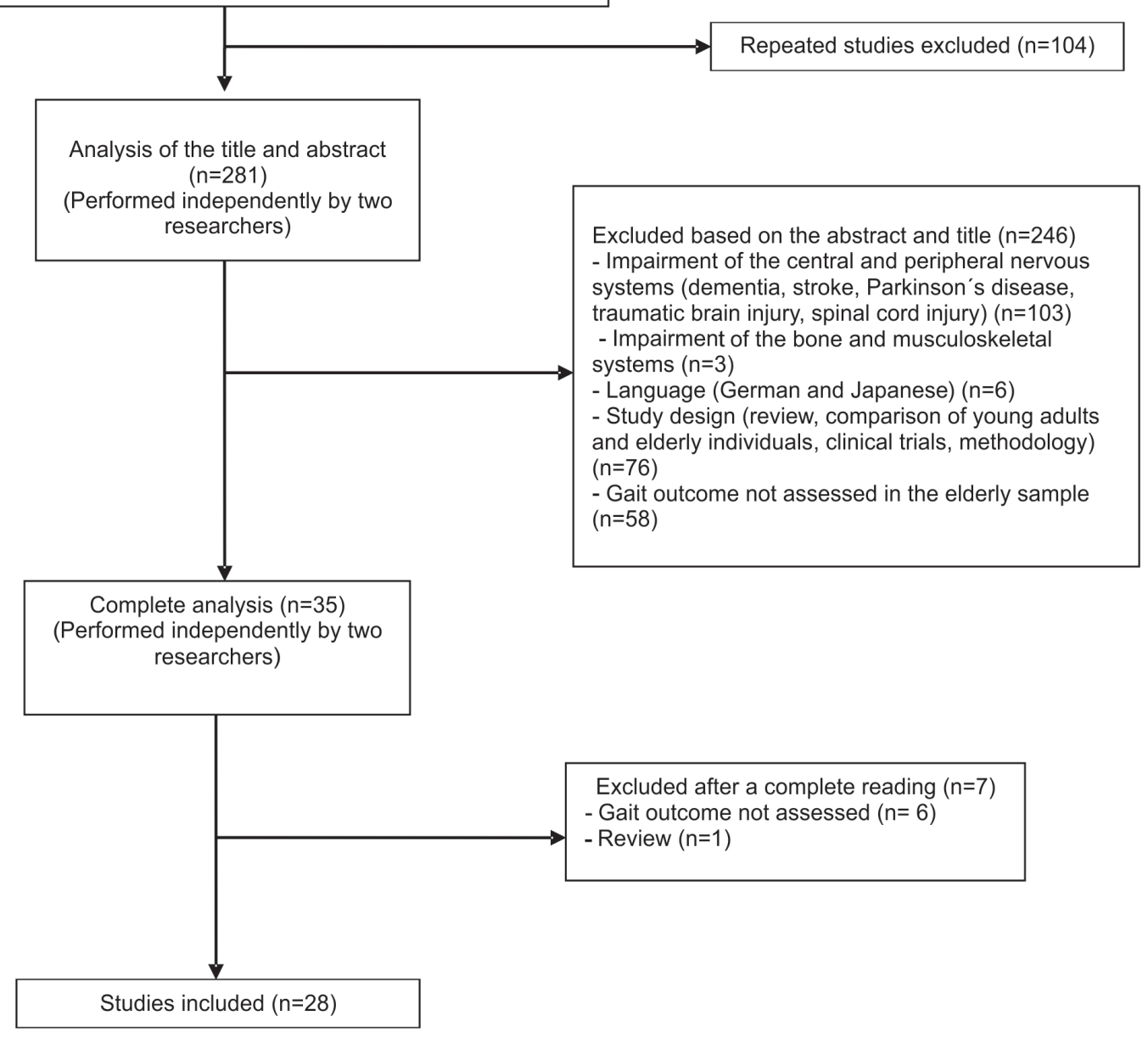

Figure 1. Fluxogram of the study selection.

All 28 of the articles included were published in English. Of these, 13 analyzed a single group of elderly individual ${ }^{25-37}$ and 15 compared groups of elderly individuals with different characteristics. ${ }^{5,6,16-21,38-44}$
Charts 1 and 2 contain the details of the studies included in this review, as well as the details of the samples, instruments, tasks and spatial and temporal parameters of gait in each study. 


\begin{tabular}{|c|c|c|c|c|c|c|c|}
\hline 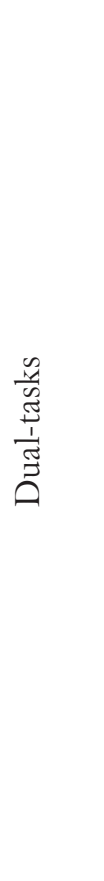 & 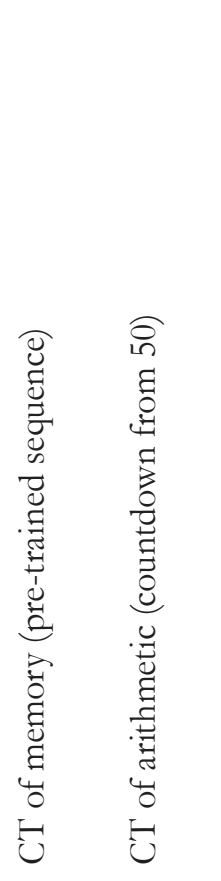 & 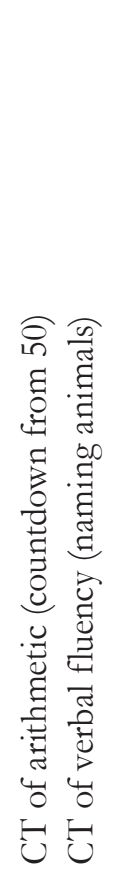 & 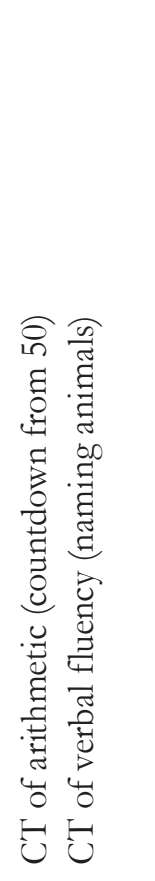 & 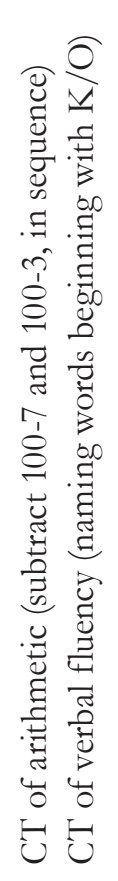 & 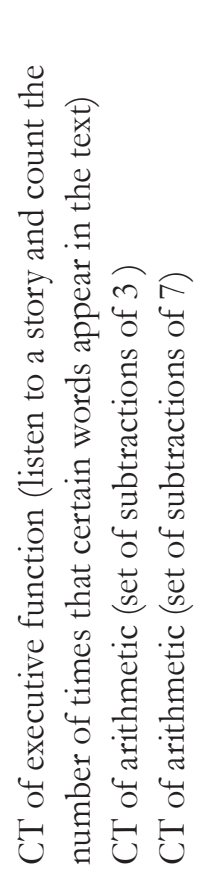 & 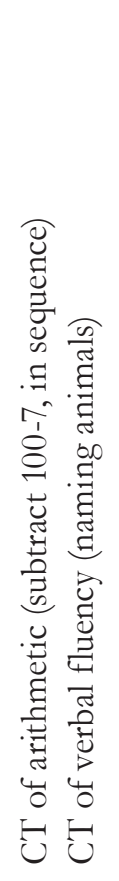 & 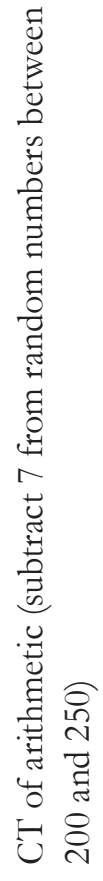 \\
\hline 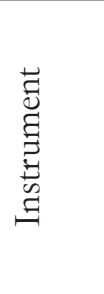 & 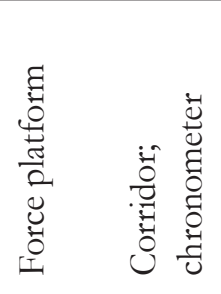 & 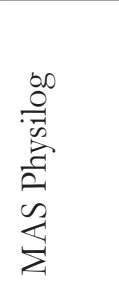 & 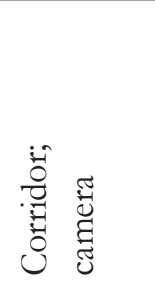 & 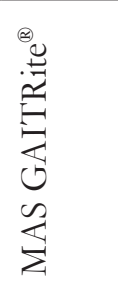 & 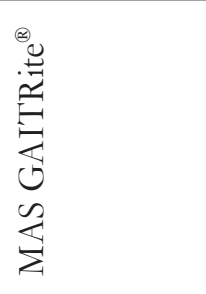 & 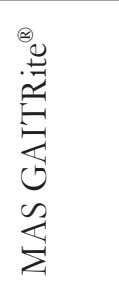 & 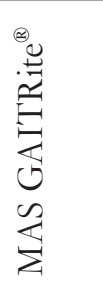 \\
\hline 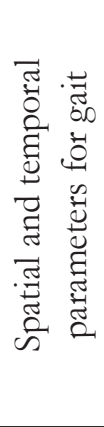 & 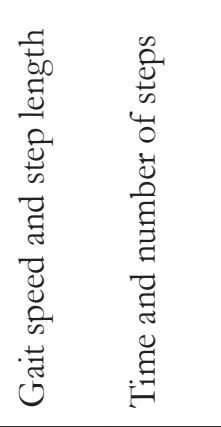 & 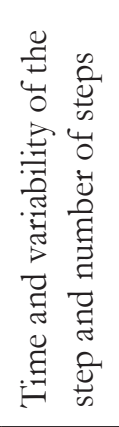 & 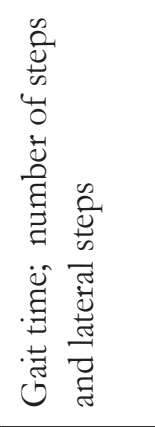 & 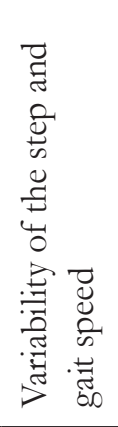 & 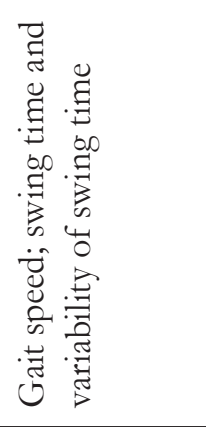 & 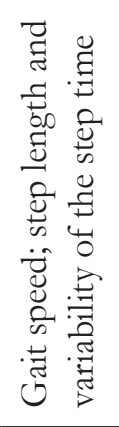 & 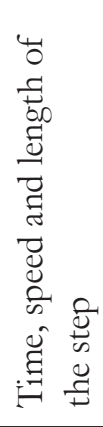 \\
\hline $\begin{array}{l}\frac{u}{\tilde{E}} \\
\text { जू }\end{array}$ & 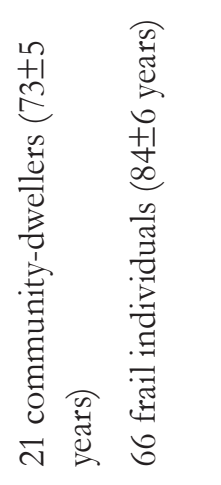 & 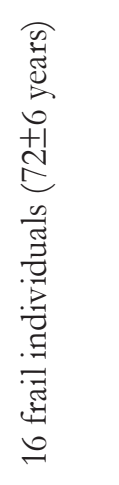 & 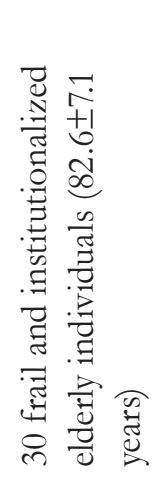 & 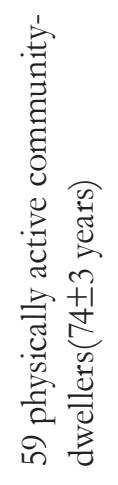 & 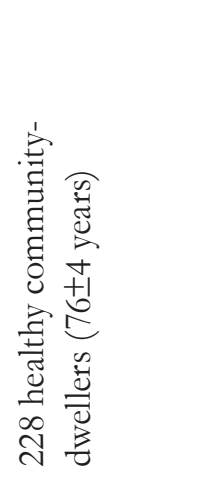 & 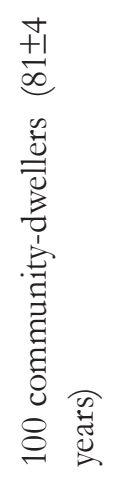 & 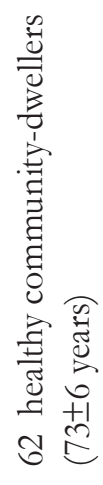 \\
\hline $\begin{array}{l}\text { 苛 } \\
\text { 营 }\end{array}$ & 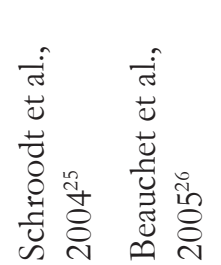 & 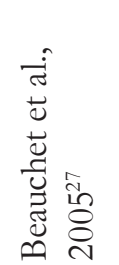 & 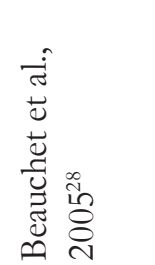 & 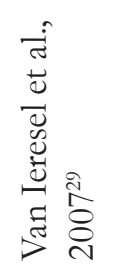 & 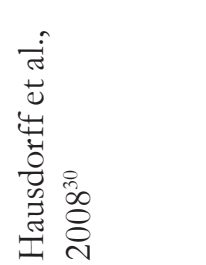 & 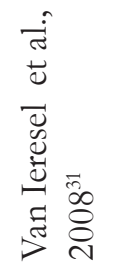 & 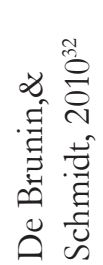 \\
\hline
\end{tabular}




\begin{tabular}{|c|c|c|c|c|c|}
\hline 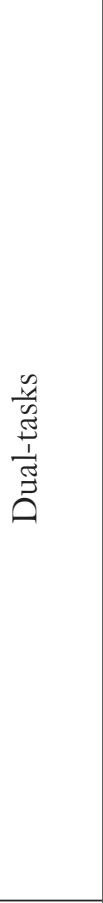 & 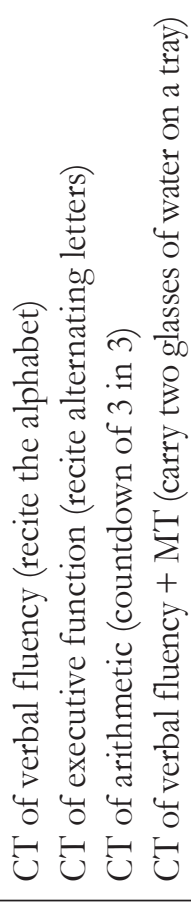 & 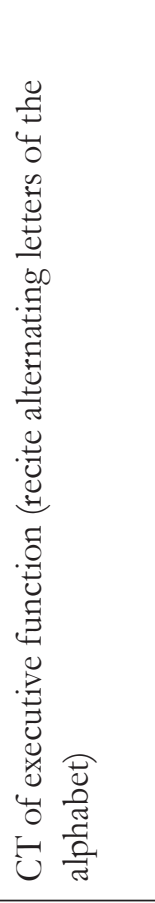 & 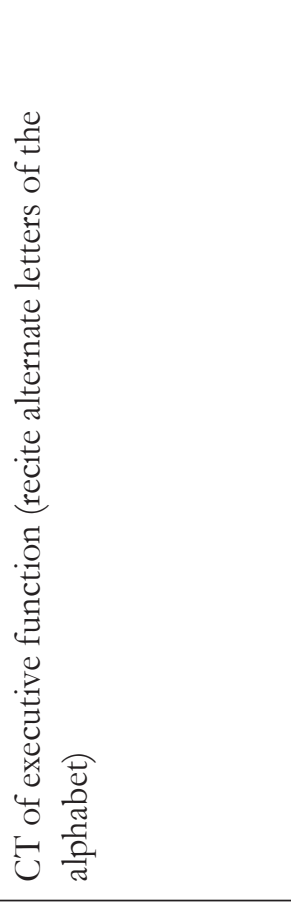 & 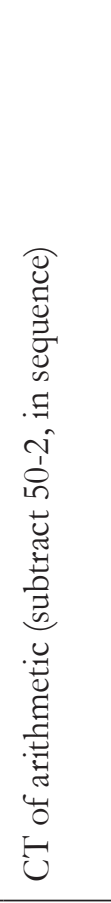 & 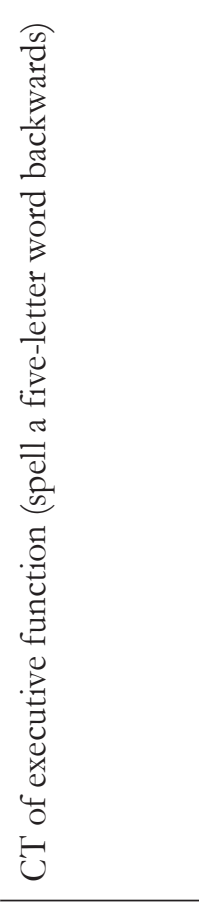 \\
\hline 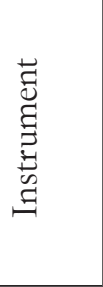 & 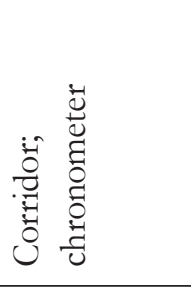 & 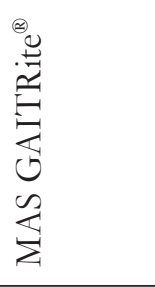 & 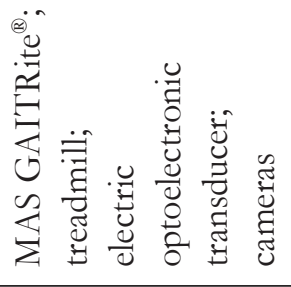 & 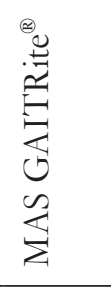 & 里总 \\
\hline 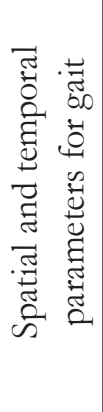 & 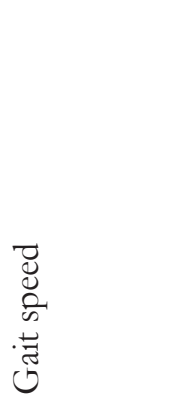 & 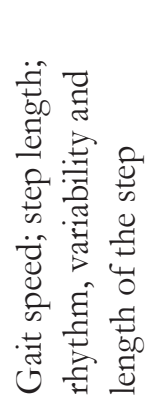 & 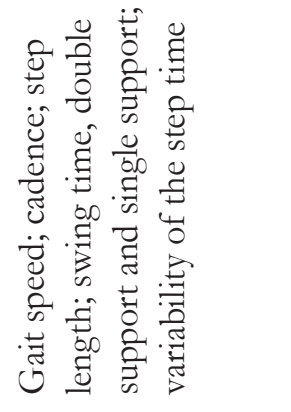 & 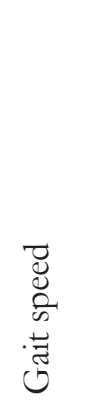 & 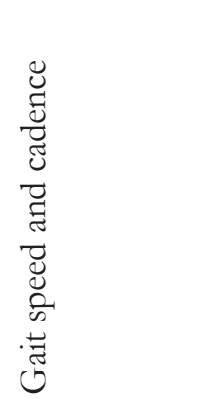 \\
\hline 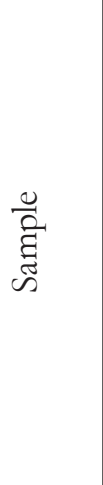 & 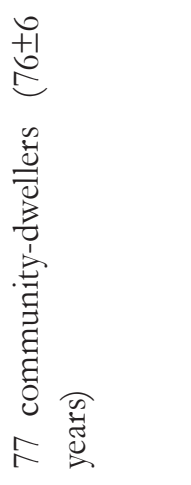 & 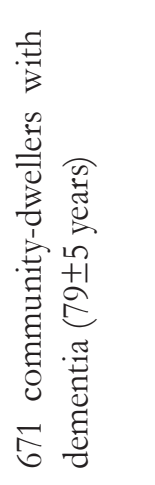 & 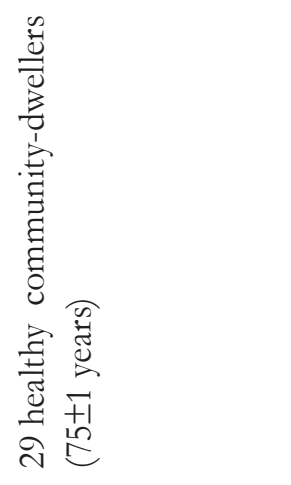 & 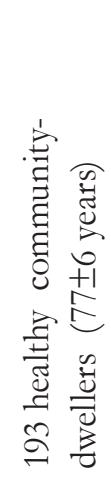 & 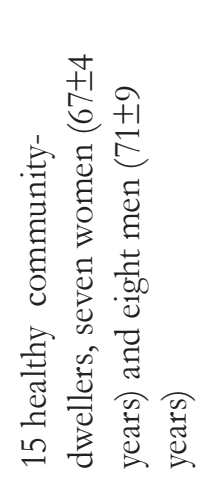 \\
\hline 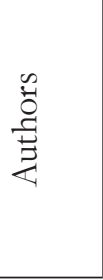 & 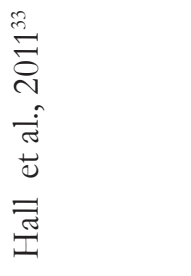 & 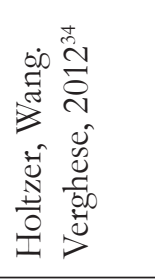 & 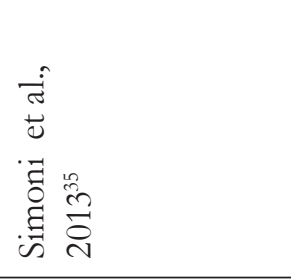 & 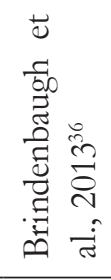 & 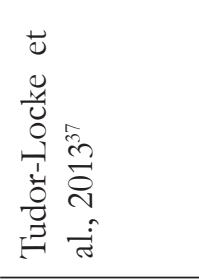 \\
\hline
\end{tabular}




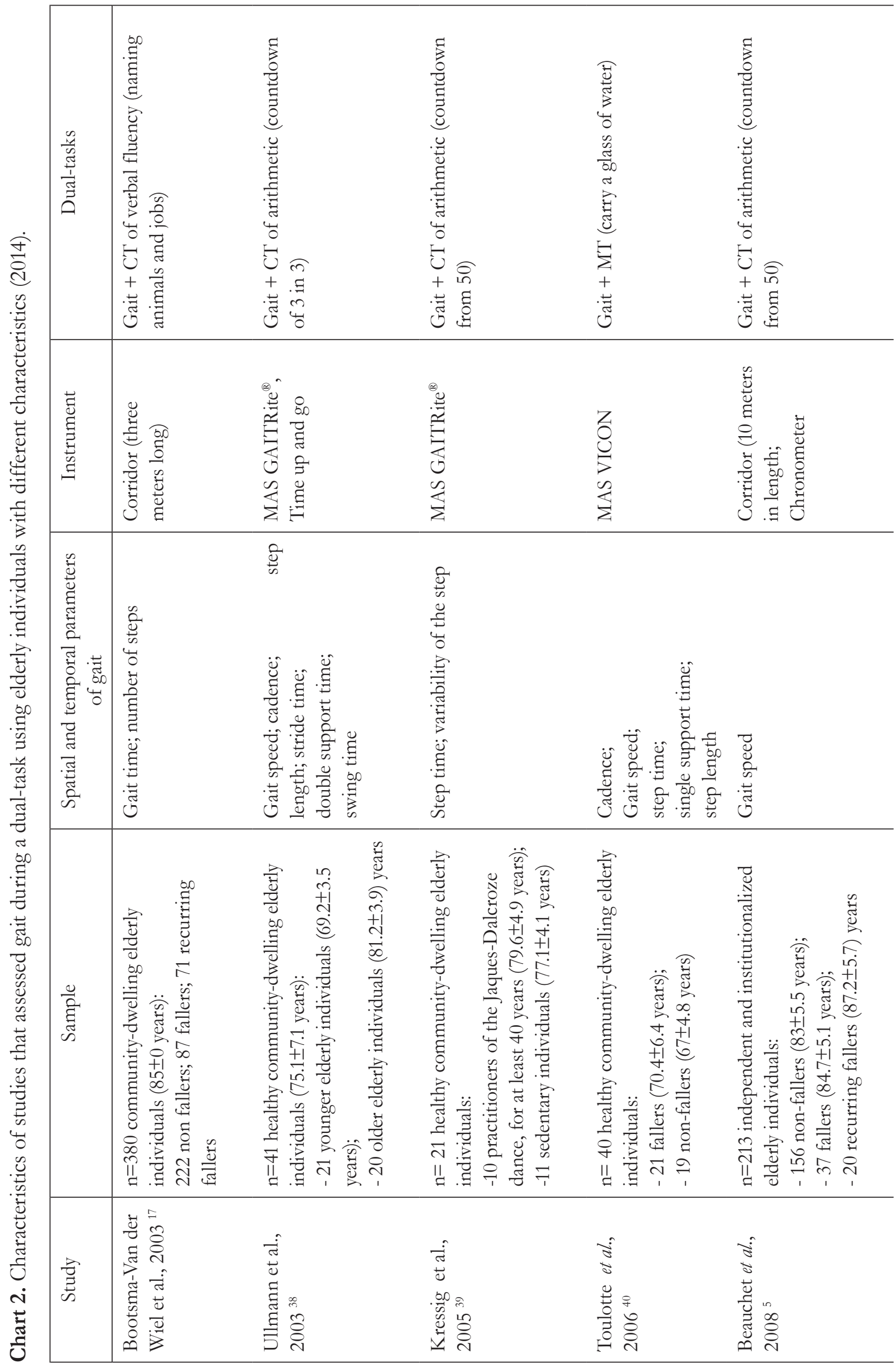




\begin{tabular}{|c|c|c|c|c|c|}
\hline 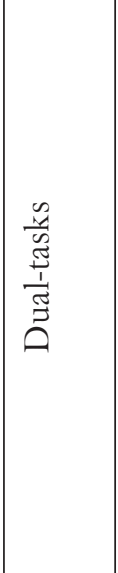 & 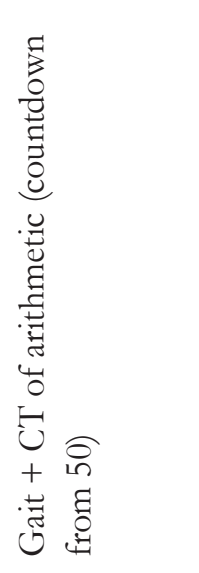 & 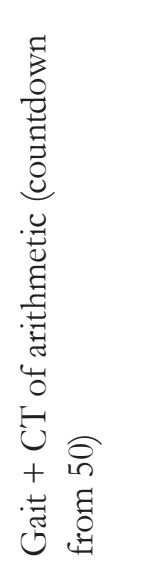 & 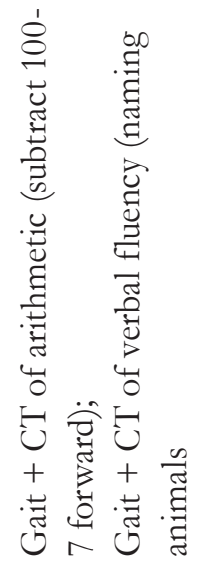 & 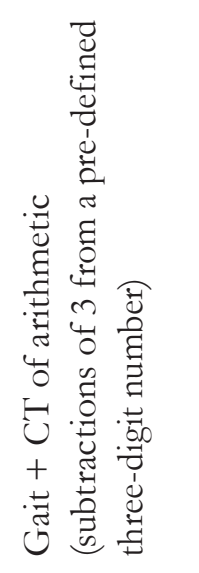 & 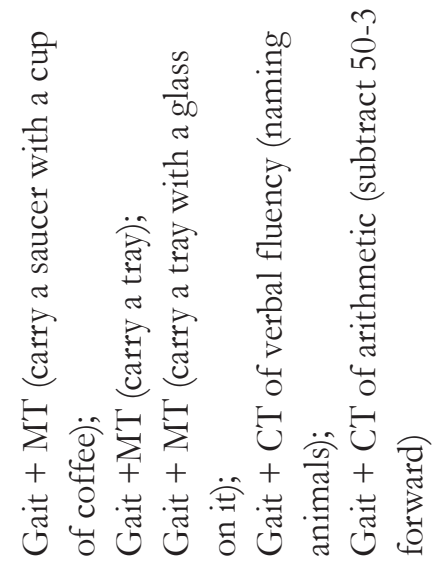 \\
\hline 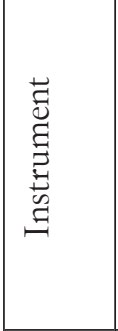 & 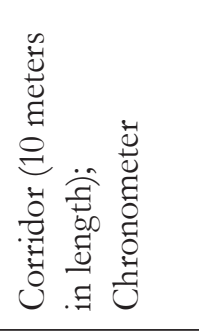 & 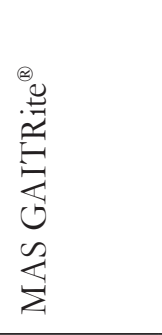 & 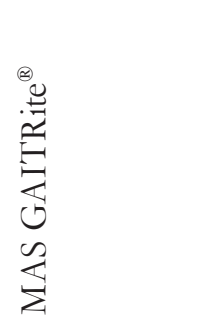 & 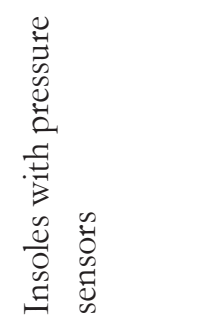 & 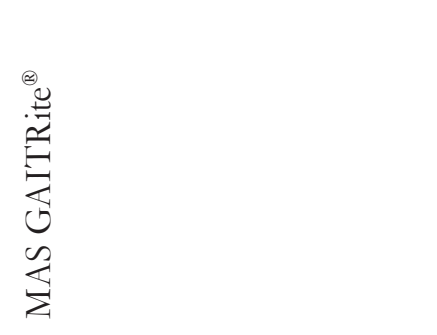 \\
\hline 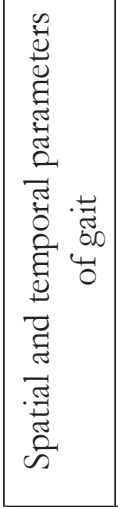 & 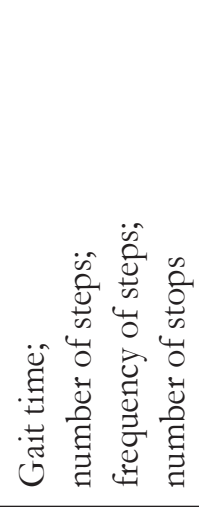 & 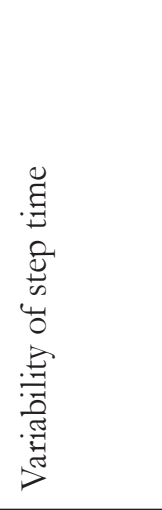 & 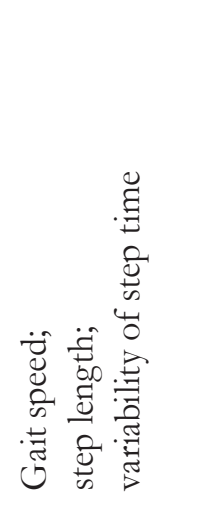 & 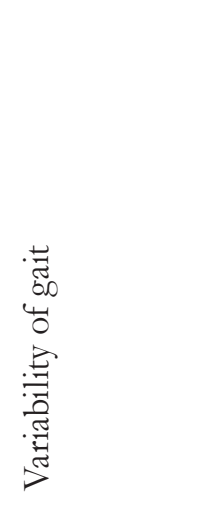 & 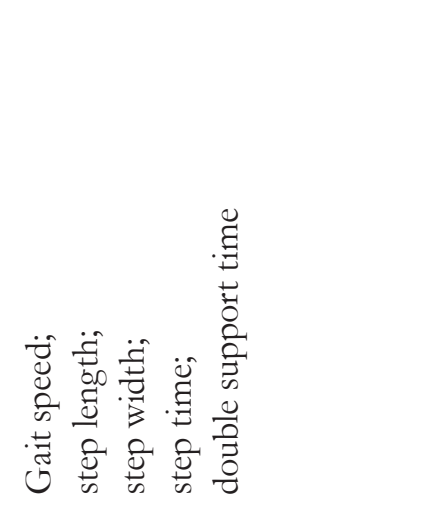 \\
\hline 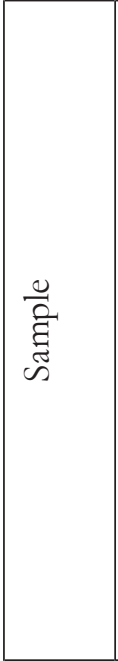 & 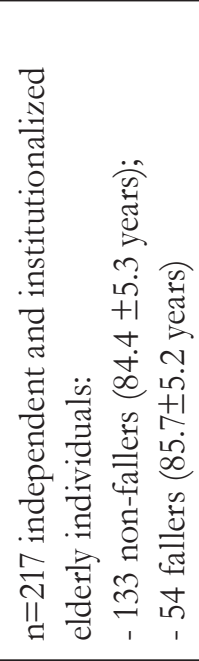 & 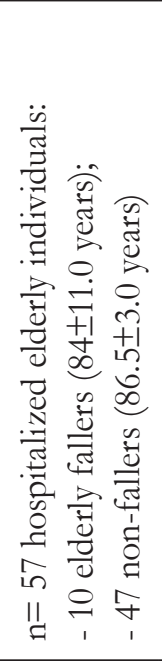 & 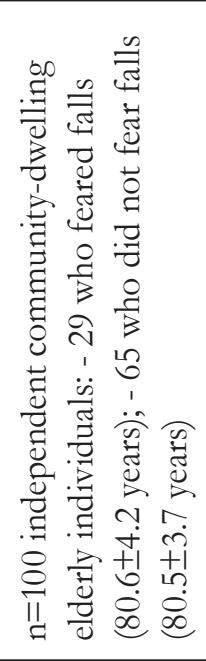 & 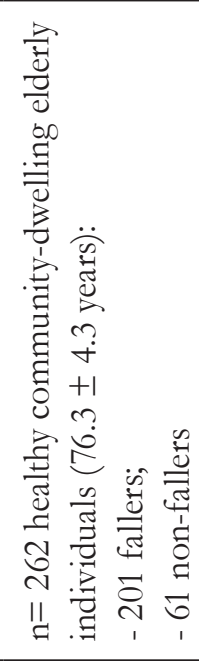 & 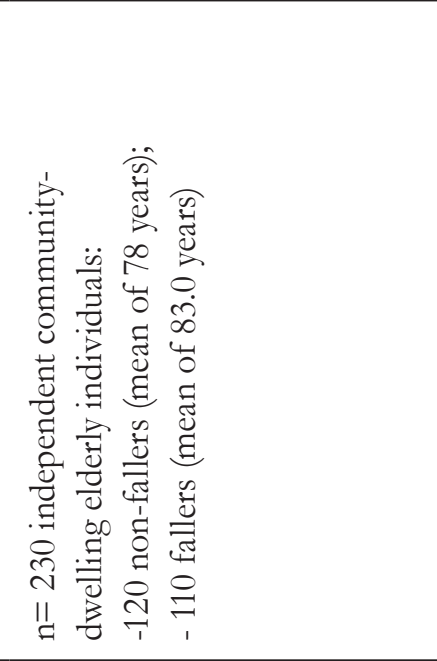 \\
\hline $\begin{array}{l}\text { Dे } \\
\text { D }\end{array}$ & 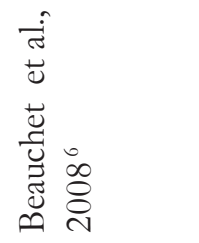 & 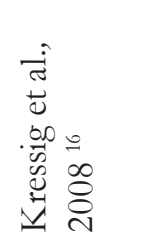 & 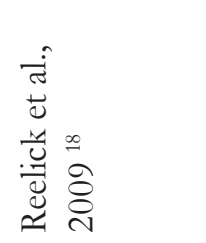 & 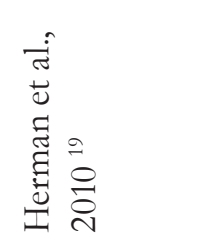 & 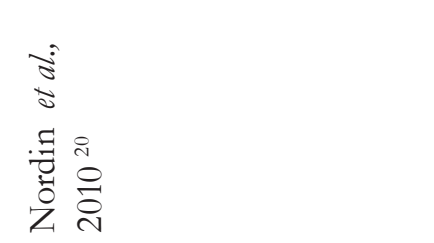 \\
\hline
\end{tabular}




\begin{tabular}{|c|c|c|c|c|}
\hline 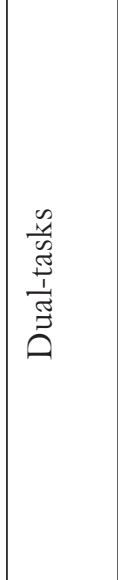 & 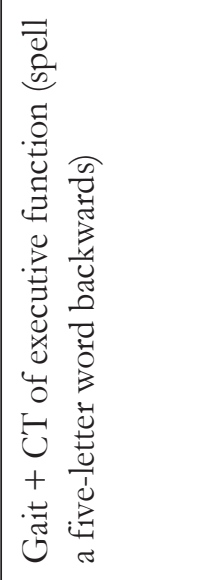 & 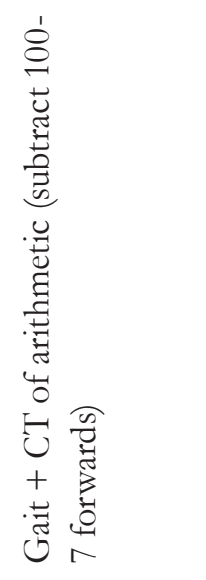 & 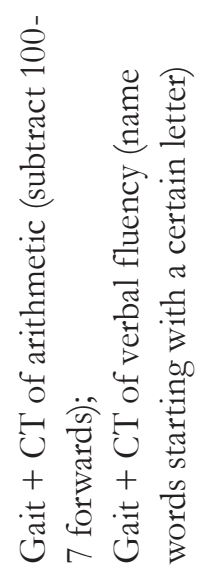 & 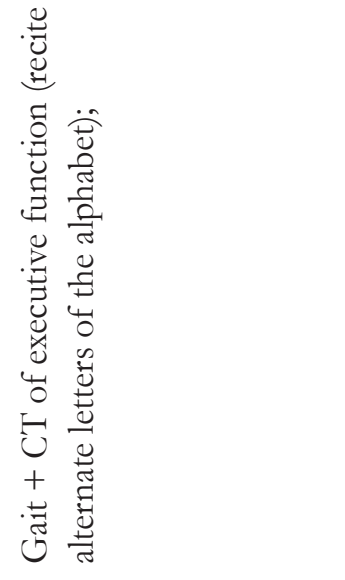 \\
\hline 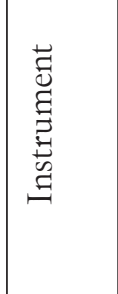 & 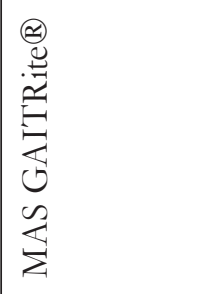 & 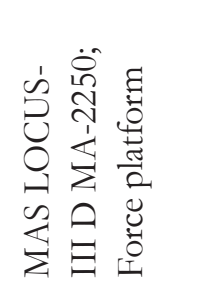 & 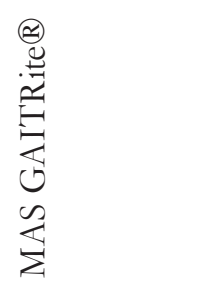 & 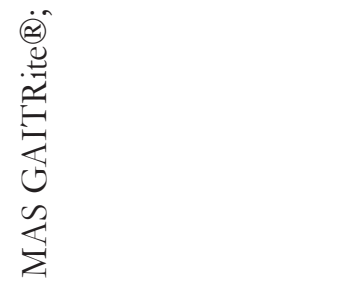 \\
\hline 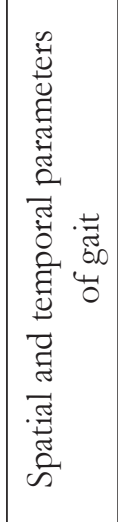 & 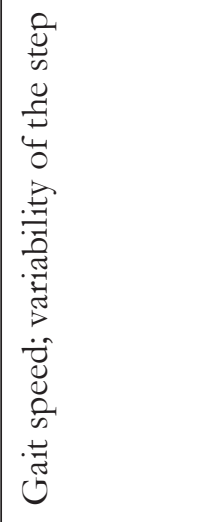 & 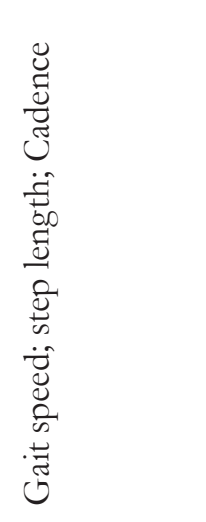 & 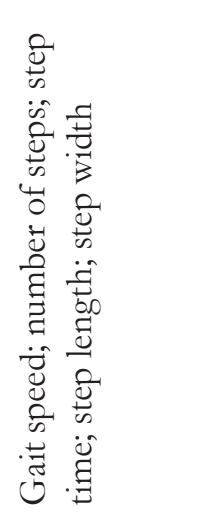 & 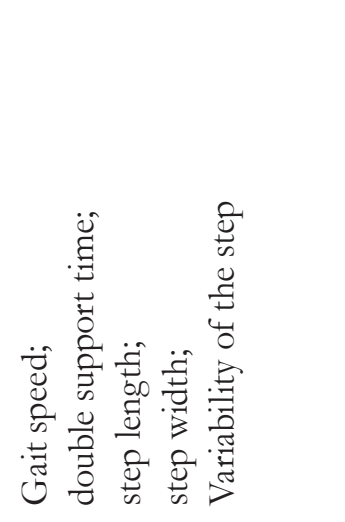 \\
\hline 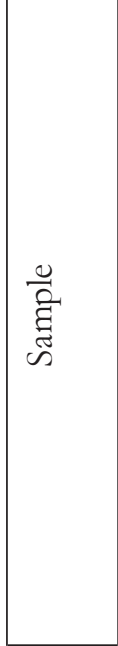 & 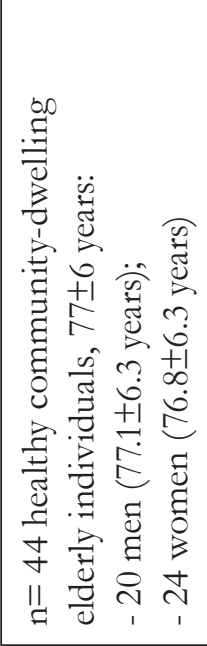 & 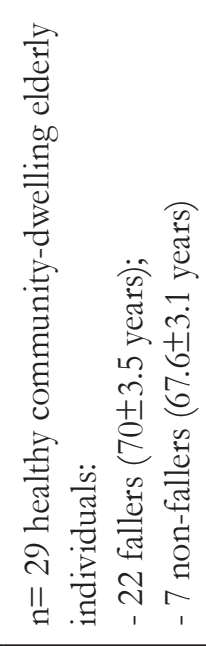 & 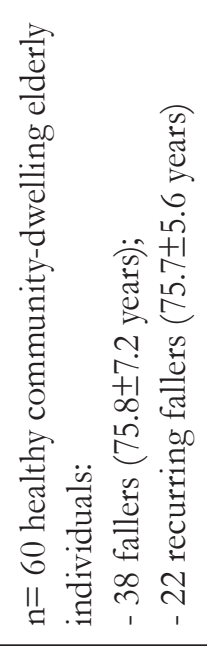 & 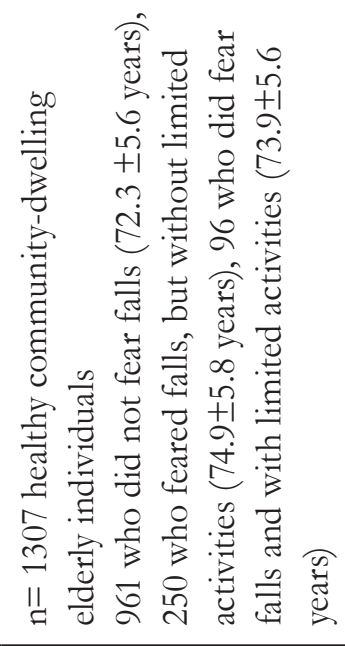 \\
\hline $\begin{array}{l}\vec{E} \\
\vec{D} \\
\stackrel{D}{n}\end{array}$ & 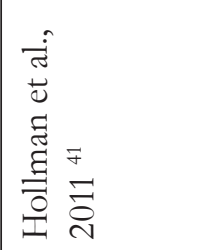 & 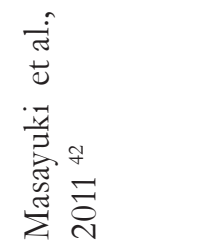 & 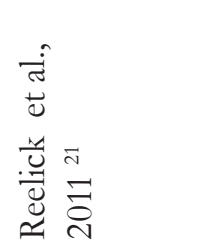 & 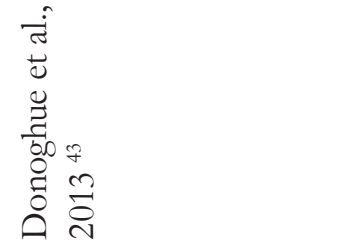 \\
\hline
\end{tabular}




\begin{tabular}{|c|c|}
\hline 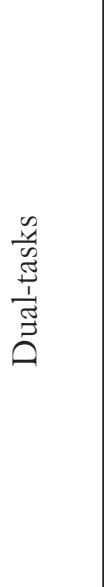 & 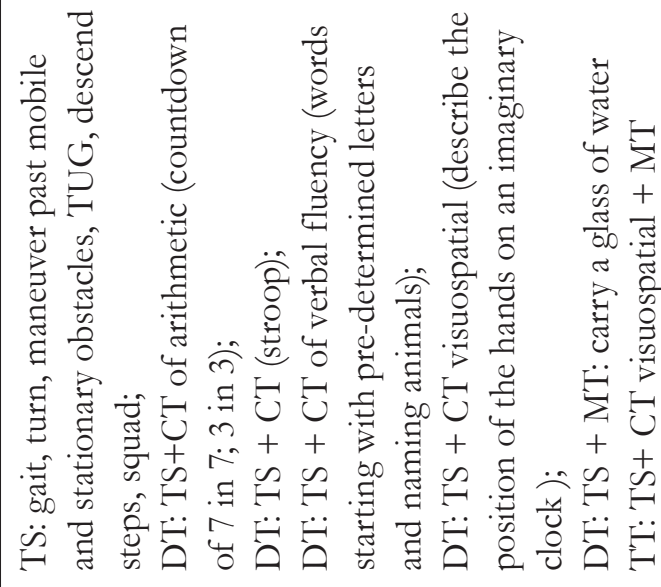 \\
\hline 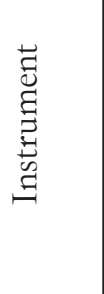 & 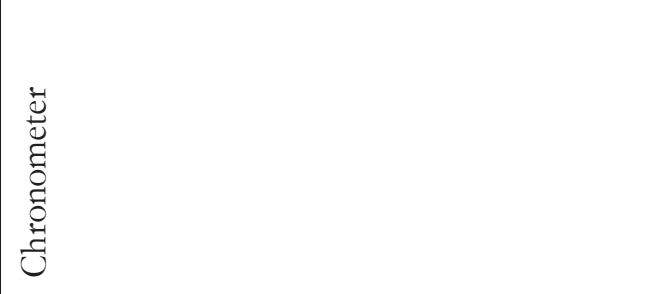 \\
\hline 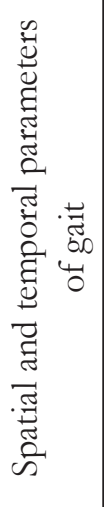 & 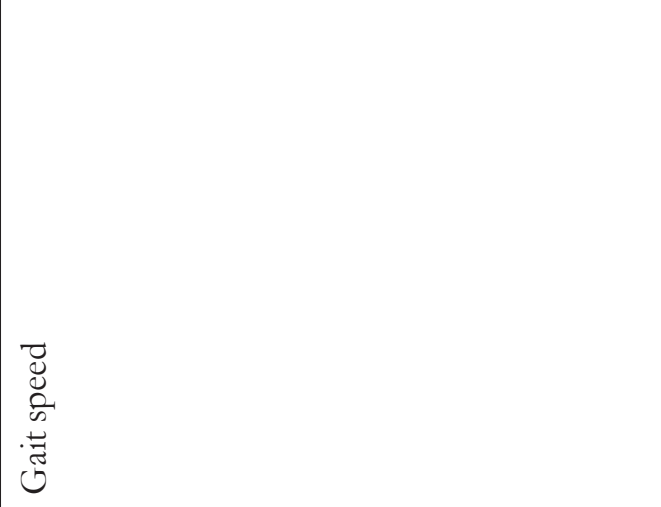 \\
\hline 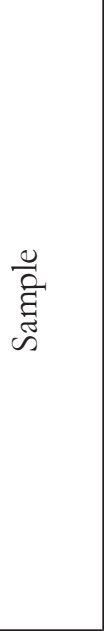 & 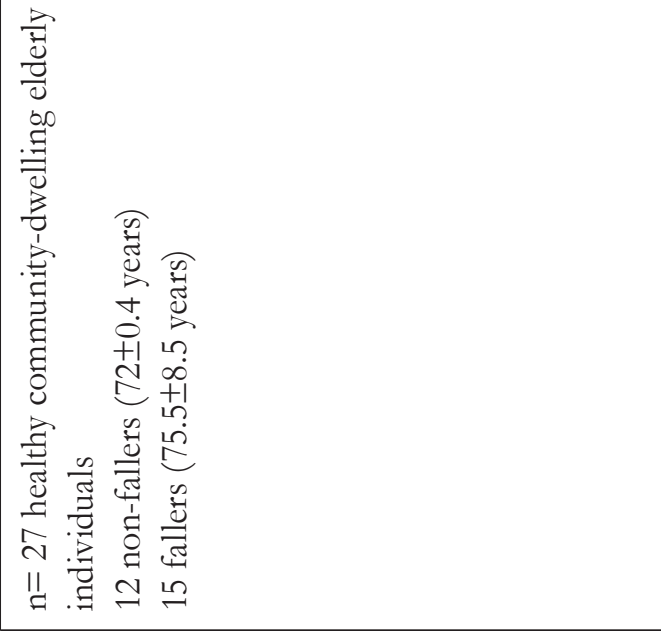 \\
\hline $\begin{array}{l}\vec{E} \\
\vec{D} \\
\stackrel{D}{n}\end{array}$ & 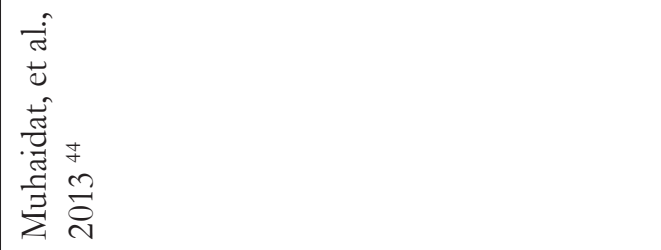 \\
\hline
\end{tabular}




\section{Spatial and temporal parameters of gait}

The spatial and temporal parameters of gait that were most often assessed were correlated with speed, stride, step, time of the gait phases and cadence.

\section{Speed}

Of the 28 studies, 19 used gait speed as the outcome. Of the nine studies that used a sample of elderly individuals with similar characteristics, eight reported a reduction in gait speed during the performance of a DT with an arithmetic CT, a verbal fluency $\mathrm{CT}$, an executive function $\mathrm{CT}$ and a triple task (TT) among frail communitydwelling elderly individuals. ${ }^{17,29-31,33-35,37}$ One study reported no alteration in the parameter during the DT. ${ }^{25}$ Of the 10 studies that compared two groups of elderly individuals with different characteristics, six indicated a reduction in gait speed among fallers, ${ }^{31,42,44}$ recurrent fallers ${ }^{5}$ and those who feared falling ${ }^{18,43}$ during a DT with an arithmetic CT, a verbal fluency CT, a motor task (MT) and a TT. The other four studies used a DT with an arithmetic CT, a verbal fluency $\mathrm{CT}$ and an executive function $\mathrm{CT}$ and found no significant effect on gait speed. ${ }^{20,21,38,41}$

\section{Stride}

Of the 28 studies, 15 assessed stride parameters. Five assessed variability and confirmed a significant increase during gait in association with a DT and an arithmetic CT, a verbal fluency $\mathrm{C} T$ and an executive function CT among elderly individuals who feared falls, ${ }^{43}$ were frail ${ }^{27}$ and lived in a community. $29,39,41$

Ten studies analyzed the stride time. Of the three studies involving elderly individuals with similar characteristics, two reported a reduction in the stride time among community-dwelling and frail elderly individuals, ${ }^{27,32}$ whereas two other studies reported an increase in the variability of time during a DT with an arithmetic CT, a verbal fluency $\mathrm{CT}$ and an executive function $\mathrm{CT} .{ }^{31,35} \mathrm{Of}$ the six studies that compared elderly individuals with different characteristics, ${ }^{16,18,38,39,40,42}$ three reported an increase in the stride time among elderly individuals who were classified as sedentary, fallers and those who feared falling, during a DT with an arithmetic CT and a MT. ${ }^{18,39,40}$

Nine studies analyzed the step length of community-dwelling elderly individuals. Of the four studies involving elderly individuals with similar characteristics, ${ }^{31-33,35}$ two reported an increase in the variability of step length ${ }^{31,33}$ and two reported a reduction of the same parameter during a DT with an arithmetic CT and a verbal fluency CT. ${ }^{32,35}$ Of the four studies that compared two groups of elderly individuals with different characteristics, ${ }^{18,21,38,40,43}$ two reported an increase in the step length during a DT with an arithmetic CT among elderly fallers and those who feared falls. ${ }^{18,21}$

One exclusive study of community-dwelling elderly individuals assessed stride speed and reported an increase during a DT with an arithmetic CT. ${ }^{32}$ Only one study assessed step width, and no statistically significant differences were found among elderly individuals that were classified as healthy, fallers and recurrent fallers during a DT with an arithmetic CT. ${ }^{21}$

Step

Twelve studies assessed step parameters and of these, six assessed the quantity of steps. Three studies only included elderly individuals with similar characteristics and reported an increase in the number of steps during a DT with an arithmetic $\mathrm{CT}$ and a verbal fluency $\mathrm{CT}$ among frail elderly individuals. ${ }^{26-28}$ Of the three studies that compared elderly individuals with different characteristics, ${ }^{5,17,21}$ two reported an increase during a DT with an arithmetic CT and a verbal fluency CT among elderly individuals classified as nonfallers, fallers and recurrent fallers. ${ }^{5,17}$

One study assessed the number of lateral steps and reported an increase for this variable during 
a DT with an arithmetic CT, but not during a DT with a verbal fluency CT, among frail and institutionalized elderly individuals. ${ }^{28}$

Two articles analyzed step width ${ }^{19,43}$ but only one reported an increase in this parameter among elderly fallers during a DT with an MT, an arithmetic CT and a verbal fluency CT, when compared with non-fallers. ${ }^{20}$

Of the five studies that assessed step length, two only analyzed elderly individuals with similar characteristics ${ }^{25,35}$ and one reported a reduction during a DT with an executive function CT among community-dwelling elderly individuals. ${ }^{35}$ Of the three studies that compared elderly individuals with different characteristics, ${ }^{20,40,42}$ two reported an increase during a DT with an MT among elderly fallers. ${ }^{20,40}$ The other study reported a reduction during a DT with an arithmetic CT among elderly fallers and non-fallers. ${ }^{42}$

Two studies assessed step time and reported an increase for this variable during a DT with an $\mathrm{MT}$, an arithmetic CT and a verbal fluency $\mathrm{CT}$ among elderly fallers. ${ }^{20,40}$

\section{Gait phases}

Six studies assessed gait phases. Of the three that assessed swing time,$^{30,35,38}$ two reported a significant reduction during the performance of a DT with an executive function CT among elderly community-dwellers. ${ }^{30,35}$ The two studies that analyzed single support time reported a significant reduction during a DT with an MT and an executive function $\mathrm{CT}$ among healthy elderly individuals and fallers. ${ }^{35,40}$ Finally, four studies investigated double support time..$^{20,35,38,43}$ Two of these studies reported a significant increase during a DT with an executive function CT and an MT among elderly community-dwellers. ${ }^{35,43}$

\section{Cadence}

Of the seven studies than analyzed cadence, ${ }^{6,33,35,37,38,40,42}$ three reported a significant increase during a DT with an MT, an arithmetic $\mathrm{CT}$ and an executive function CT among elderly individuals classified as healthy, fallers and nonfalllers. ${ }^{37,40,42}$ Two other studies reported a reduction during a DT with a verbal fluency CT and an executive function $\mathrm{CT}$ among community-dwelling elderly individuals. ${ }^{33,35}$ The two final studies of these seven reported a significant difference between "younger" and "older" elderly individuals for this parameter, ${ }^{38}$ as well as between non-fallers and fallers during a DT with an arithmetic CT. ${ }^{6}$

\section{Dual tasks and instruments used}

Of the secondary tasks used, an arithmetic CT was the most common, followed by a verbal fluency CT. Figure 2 displays the frequency of use of the tasks in the studies included in this review. Several types of instruments were used to assess the spatial and temporal parameters of gait, including very simple methods, such as a demarcated corridor and a chronometer, ${ }^{5,6,16,26,28,33,17}$ and more complex motion analysis systems (MAS), ${ }^{17-21,25,27,29-35,37-41}$ as can be seen in Figure 3 . 


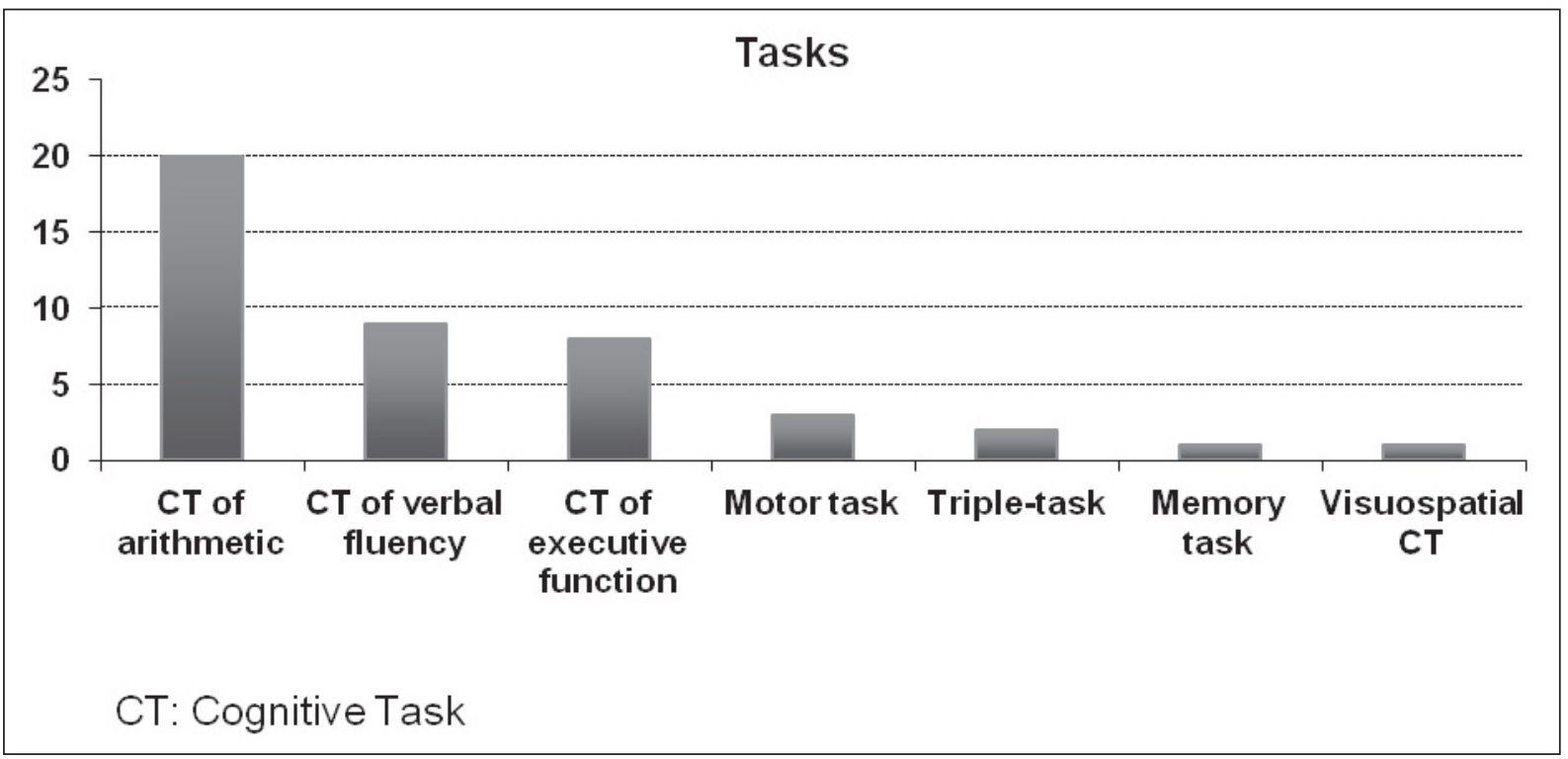

Figure 2. Frequency of tasks used to assess gait during the dual-task.

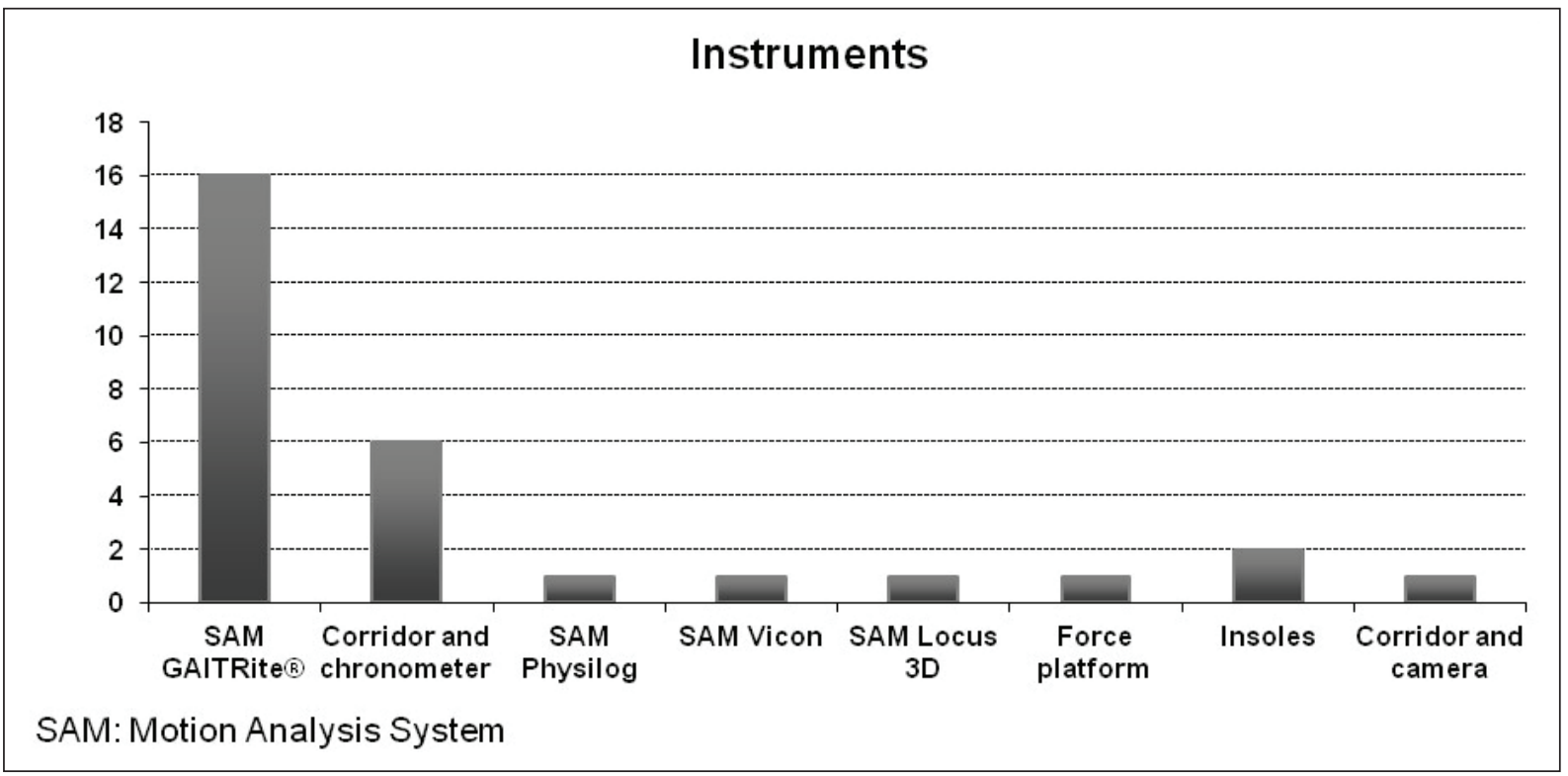

Figure 3. Frequency of instruments used to assess gait during the dual-task.

\section{DISCUSSION}

Based on the results of this review, the execution of a DT during gait altered the spatial and temporal parameters by reducing the speed and increasing the variability of the stride and the double support time. As the difficulty of the task increased, the alterations in the gait parameters increased. For example, those that required greater resources of executive function led to greater modifications. Concerning the instruments used in the analysis, motion analysis systems were the most common, followed by a chronometer and a demarcated corridor. Gait speed was the most commonly used measurement. 


\section{Spatial and temporal parameters of gait}

Currently, speed is the most commonly used measurement when discussing gait ${ }^{33}$ and it was the most commonly analyzed parameter in the studies included in this review. The assessment of gait speed during a DT is fast and easy to perform, with no sophisticated equipment required, which favors its use in clinical contexts. ${ }^{5,6}$ Studies have shown that gait speed is heavily influenced by a DT, ${ }^{5,6}$ which was corroborated by most of the results of the studies selected for this review. Many studies have reported a reduction in this parameter during a DT with an arithmetic CT, a verbal fluency CT, an executive function $\mathrm{CT}$, a triple-task or a motor task among elderly individuals classified as frail, community-dwellers, fallers, recurrent fallers and those who fear falls. $5,18,23,24,29-31,33,35,40,42$ According to Hollman et al. ${ }^{23}$ and Reelick et al., ${ }^{18}$ a reduction in gait speed is not necessarily a predictor of gait instability or a risk factor for falls, although it could indicate a fear of falls or postural control disorders. A reduction in gait speed could be seen as a compensation mechanism during gait, when its stability is challenged, in order to minimize the destabilization of postural control. ${ }^{18,29}$

Stride variability was also affected during gait with a DT, exhibiting a significant increase in most of the studies analyzed. ${ }^{27,29,39,41}$ The increase in variability from one step to another reflects the variability in the length, time and consequently, the speed of the steps, which hinders the individual's ability to regulate step variations in the gait time. ${ }^{23}$ Thus, it is possible to infer that the increase in step variability could reflect gait instability. ${ }^{23,24}$ According to Hausdorff et al., ${ }^{30}$ the variability of the length of the step is a better fall predictor than gait speed. ${ }^{9}$ According to Reelick et al., ${ }^{18}$ increases in the variability of step time and step length are correlated with a fear of falls, although this correlation was explained by the alteration in gait speed. ${ }^{18}$

It has been reported that elderly fallers and non-fallers exhibit significant differences in gait parameters during a DT. ${ }^{5,6,17,19,20,40}$, It is possible that the structure and function of the motor-sensory system are more impaired among those classified as fallers, who exhibit less capacity to adapt during the performance of a DT. This leads to modifications in the gait parameters and favors falls. ${ }^{40}$

Two studies reported an increase of cadence during a DT ${ }^{40,42}$ and two other studies reported a reduction in cadence during a DT. ${ }^{33,35}$ One possible explanation for this is the type of secondary task used. The studies that reported an increase in cadence used an MT and an arithmetic CT, whereas those that reported a reduction used a verbal fluency CT and an executive function CT. These results show that motor function tasks demand more care and motor coordination. In the case of a verbal fluency CT, the rhythm required to respond may lead to a regulator effect for cadence. ${ }^{45}$ Another factor was the heterogeneity of the sample: the studies that reported an increase in cadence included healthy elderly individuals and fallers; the studies that reported a reduction only contained healthy elderly individuals. It is also possible that the arithmetic task demanded greater care than the verbal fluency task, causing more disturbance during the performance of the DT.

There is a certain amount of controversy linked to correlations between cadence, step length and gait speed, with several studies reporting a direct correlation between these parameters ${ }^{46}$ and others disagreeing, suggesting that there are different control mechanisms for cadence and speed. ${ }^{33,42}$ Gait speed and step length are probably controlled by the cortical-subcortical circuit, through the thalamus, whereas cadence is controlled by mechanisms of the brainstem and spinal cord. ${ }^{33,46}$

\section{Dual-tasks}

One question that is significant in clinical practice is which cognitive task to use when assessing the performance of dual-tasks. The answer depends on the cognitive and motor state of the individual. ${ }^{33}$ Concerning the secondary tasks used in the majority of the studies, an arithmetic CT resulted in a greater step variability than a verbal fluency CT. ${ }^{27,28}$ This difference could be due to the distinct attentional load associated with these two cognitive tasks. Since the person's 
attention is split between two simultaneous tasks, their performance will depend on the type of tasks and their difficulty level. ${ }^{20,27,30}$ This means that alterations in gait that are correlated with a DT increase in line with an increase in the difficulty of the task.

While a verbal fluency task depends on semantic memory, an arithmetic task essentially depends on working memory (a temporary storage and data processing system), which is directly associated with executive functions. ${ }^{20,27,28}$ Therefore, the competition for executive functions that, in a DT situation, are used to coordinate the two tasks, has a greater effect during an arithmetic CT than a verbal fluency CT, due to the greater variability observed during gait with an arithmetic CT. ${ }^{27,28}$ Beauchet et al. ${ }^{6}$ found a strong correlation between the performance of an arithmetic CT as a secondary task to gait and the first fall among elderly non-fallers. ${ }^{6}$ In addition, attention and executive function were the most important cognitive functions in the regulation of gait and the control of balance among elderly individuals. This factor has shown a direct correlation between cognitive function, gait speed and falls., $5,626-29,33$

Concerning frail individuals, studies have shown that the effects of a DT on gait parameters are correlated with the occurrence of falls. ${ }^{5,6,28}$ One considerable alteration is demonstrated by the instability in the medial-lateral direction, which was clear from the increase in the number of lateral steps taken during the performance of an arithmetic CT, when compared with a verbal fluency CT. This could indicate a strong predictor of falls. ${ }^{6,28}$ Thus, the competitive interaction between tasks leads to interference between them, which explains the more significant lateral instability during an arithmetic CT (correlated with executive function), when compared with a verbal fluency CT (not directly correlated with executive function) ${ }^{28}$

Furthermore, when comparing two types of cognitive tasks, an arithmetic CT with a countdown is a more rhythmic task than a verbal fluency CT. When the elderly individual performs a countdown task simultaneously during gait, one task interferes with the other, and the brain tends to equalize the rhythm of both. The characteristic rhythm of the countdown could symbolize a walking rhythm, thereby regulating the step pattern. ${ }^{45}$

Unlike these studies, in which gait was directly affected by an arithmetic CT and a verbal fluency CT, other studies have reported a similar effect, regardless of the type of secondary task used (arithmetic or verbal fluency). ${ }^{18,29,31,33}$ One explanation for these contradictory results could be the use of different strategies by the participants, for different $\mathrm{DT},{ }^{21}$ such as prioritizing one of the two tasks.

It has been reported that the performance of a secondary MT during gait led to a reduction in cadence, speed, step and stride length, as well as an increase in the single and double support times among elderly fallers and non-fallers. ${ }^{40}$ When compared with a CT, the effect of an MT (carrying a glass) on the width, time and length of the step of elderly fallers and non-fallers was correlated with a low risk of falls, possibly due to the stabilizing nature of holding a glass: the movements of the trunk and upper limbs are restricted, leading to biomechanical adjustments to the center of the mass and subsequent adjustments to the support base. ${ }^{20}$

Instruments

In terms of the techniques used to measure the kinematic gait parameters, a corridor and chronometer combination was the most simple methodology and the second most commonly used in studies that assessed gait during a DT, providing data on parameters such as speed and step frequency. ${ }^{18,23,24}$ The advantages of this type of instrumentation include ease of access, easy clinical applicability and reliability in assessing gait speed. ${ }^{44,47,48}$

For MAS, carpets with pressure sensors were the most commonly used instruments to assess the kinematic parameters of gait during a DT. One possible explanation for this is the variety of parameters that can be recorded by this system, including speed, step and stride variables, cadence, support base, the distribution of plantar 
pressure, the angle of foot progression and the displacement from the pressure center. In addition, the GaitRite ${ }^{\circledR}$ carpet with pressure sensors (the most commonly used in the studies of this review) has been validated and exhibits adequate reliability for assessments of most spatial and temporal parameters related to gait for both young and old populations, with a reliability index that ranges from 0.82 to 0.91 between examiners. ${ }^{22,49}$

Concerning the quantity of steps needed to obtain reliable estimates of the parameters assessed by the carpets with pressure sensors, Beeser et al. ${ }^{50}$ reported that five to eight steps are needed for speed, step length and single support time, whereas more than 10 steps are required for reliable data related to other parameters, such as the support base width and double support time. Studies have shown that three attempts with a validated instrument will provide reliable measurements for speed and cadence, although this is not sufficient for the variability of step speed, which requires the analysis of a greater number of steps. ${ }^{24}$ Reelick et al. ${ }^{21}$ suggested that there are no significant differences in the variability of the gait parameters of elderly fallers and recurrent fallers, due to the limited number of steps used. Thus, the instrument to be selected should be minutely analyzed and correctly used, depending on the parameters to be assessed.

The differences found between the results of some of the studies included in this review could be explained by sample size, age groups with different strata, the representativeness of the sample, the different types of instruments used in the assessments, the nature of the secondary tasks used, the prioritization (or not) of a task, and the peculiarities of the cognitive domain.

This review contains a number of limitations. The quality of the observational studies included in this review was not assessed systematically. In addition, this review did not include studies that analyzed kinetic gait parameters or those that performed three-dimensional kinetic analysis. Including these types of studies could lead to a greater contribution to clinical practice.

\section{CONCLUSION}

Elderly individuals exhibit abnormalities in the spatial and temporal parameters of gait, such as speed, step length, cadence and the time involved in gait phases, when performing a dual-task. These abnormalities tend to have more of an impact on older individuals. Gait speed and step variability were the most commonly assessed parameters in studies of the gait of elderly individuals who performed a dual-task. Together, these two parameters are considered excellent indicators of the risk of falls.

An arithmetic cognitive task was the most common secondary task in the studies selected, followed by a verbal fluency cognitive task. In addition, this review noted that as the complexity of the secondary task increased, the repercussions on the variability of the gait parameters also increased.

Different instruments were used to assess gait with a dual-task among elderly individuals. The most common instrument was a carpet with pressure sensors. The use of a corridor and chronometer was also quite common, due to their greater accessibility and applicability.

The data available is still not cohesive to recommend specific, reliable tasks for the prediction of greater impairment of postural control during gait with a dual-task. Therefore, it is correct to state that gait speed and the variability of the step best demonstrate the effect of the dual-task on dynamic postural control. However, it is still too soon to indicate which tests and instruments perform best in assessments of the spatial and temporal parameters of gait during a dual-task and assessments of the probability of falls.

The knowledge acquired through this review could contribute to a better understanding of the motor control of elderly individuals during the performance of a dual-task, as well as improving the assessments and therapeutic approaches used for individuals who exhibit abnormal gait, irregular postural control and an increased risk of falls. 


\section{REFERENCES}

1. Cruz DT, Ribeiro LC, Vieira MT, Teixeira MTB, Bastos RR, Leite ICG. Prevalência de quedas e fatores associados em idosos. Rev Saúde Pública 2012;46(1):138-46.

2. Mazo GZ, Liposcki DB, Ananda C, Prevê D. Condições de saúde, incidência de quedas e nível de atividade física dos idosos. Rev Bras Fisioter 2007;11(6):437-42.

3. Brito TA, Fernandes MH, Coqueiro RS, Jesus CS. Quedas e capacidade funcional em idosos longevos residentes em comunidade. Texto Contexto Enferm 2013;22(1):43-51.

4. Bridenbaugh SA, Kressig RW. Laboratory review: the role of gait analysis in seniors' mobility and fall prevention. Gerontology 2011;57(3):256-64.

5. Beauchet O, Annweiler C, Allali G, Berrut G, Herrmann FR, Dubost V. Recurrent falls and dual task-related decrease in walking speed: is there a relationship? J Am Geriatr Soc 2008;56(7):1265-9.

6. Beauchet O, Allali G, Annweiler C, Berrut G, Maarouf $\mathrm{N}$, Herrmann FR, et al. Does change in gait while counting backward predict the occurrence of a first fall in older adults? Gerontology 2008;54(4):217-23.

7. Woollacott M, Shumway-Cook A. Attention and control of posture and gait: a review of an emerging area of research. Gait Posture 2002;16(1):1-14.

8. Brauer CG, Woolacott M, Shumway-Cook A. The influence of a concurrent cognitive task on the compensatory stepping response to a perturbation in balance-impaired and healthy elders. Gait Posture 2002;15(1):83-93.

9. Barbosa JMM, Prates BDSS, Gonçalves CF, Aquino AR, Parentoni AN. Efeito da realização simultânea de tarefas cognitivas e motoras no desempenho funcional de idosos da comunidade. Fisioter Pesqui 2008;5(4):374-9.

10. Yogev-Seligmann G, Hausdorff JM, Gilad N. The role of executive function and attention in gait. Mov Disord 2008;23(3):329-42.

11. Moraes H, Deslandes A, Silveira H, Arcoverde C, Alve H, Laks J. Effects of a motor and cognitive dual-task performance in depressive elderly, healthy older adults, and healthy young individuals. Dement Neuropsychol 2011;5(3):198-202.

12. Torres JL. Influência da dupla tarefa nos parâmetros espaço-temporais da marcha de idosos: uma revisão da literatura [Monografia de especialização]. Minas Gerais: Universidade Federal de Minas Gerais, Escola de Educação Física, Fisioterapia e Terapia Ocupacional; 2010.

13. Gehring PR, Bertolassi MA, Nunes MES, Basso L, Meira CDM Júnior, Dos Santos S. Desempenho de idosos em uma tarefa motora de demanda de duplo controle. Rev Bras Educ Fís Esp 2009;23(3):211-20.

14. Montero-Odasso M, Bergman H, Phillips NA, Wong $\mathrm{CH}$, Sourial N, Chertkow H. Dual tasking and gait in people with mild cognitive impairment. The effect of a memory-work. BMC Geriatrics 2009;41(9):1-8.

15. Menz HB, Lord SR, Fitzpatrick RC. Agerelated differences in walk stability. Age Ageing 2003;32(2):137-42.

16. Kressig RW, Herrmann FR, Grandjean R, Michel JP, Beauchet O. Gait variability while dual-tasking: fall predictor in older inpatients? Aging Clin Exp Res 2008;20(2):123-30.

17. Bootsma-van der Wiel A, Gussekloo J, De Craen A J, Van Exel E, Bloem B R, Westendorp RG. Walking and talking as predictors of falls in the general population: the Leiden 85-Plus Study. J Am Geriatr Soc 2003;51(10):1466-71.

18. Reelick MF, Van Iersel MB, Kessels RP, Rikkert MGO. The influence of fear of falling on gait and balance in older people. Age Ageing 2009;38(4):435-40.

19. Herman T, Mirelman A, Giladi N, Schweige A, Hausdorff JM. Executive control deficits as a prodrome to falls in healthy older adults: a prospective study linking thinking, walking, and falling. J Gerontol Ser A Biol Sci Med Sci 2010;65(10):1086-92.

20. Nordin E, Moe-Nilssen R, Ramnemark A, LundinOlsson L. Changes in step-width during dual-task walking predicts falls. Gait Posture 2010;32(1):92-7.

21. Reelick MF, Kessels RP, Faes M C, Weerdesteyn V, Esselink R A, Rikkert MGO. Increased intraindividual variability in stride length and reaction time in recurrent older fallers. Aging Clin Exp Res 2011;23(5-6):393-9.

22. Menz HB, Latt MD, Tiedemann A, Mun San Kwan M, Lord SR. Reliability of the GAITRite ${ }^{\circledR}$ walkway system for the quantification of temporo-spatial parameters of gait in young and older people. Gait Posture 2004;20(1):20-5

23. Hollman JH, Salamon KB, Priest AW. Age-related differences in stride-to-stride variability during dual task walking: a pilot study. J Geriatr Phys Ther 2004;27(3):83-7.

24. Priest AW, Salamon KB, Hollman JH. Age-related differences in dual task walking: a cross sectional study. J Neuroengineering Rehabil 2008;5:1-8.

25. Schroodt LA, Mercer VS, Giuliani CA, Hartman M. Characteristics of stepping over an obstacle in community dwelling older adults under dual-task conditions. Gait Posture 2004;19(3):279-87. 
26. Beauchet O, Dubost V, Herrmann F, Rabilloud M, Gonthier R, Kressig RW. Relationship between dualtask related gait changes and intrinsic risk factors for falls among transitional frail older adults. Aging Clin Exp Res 2005;17(4):270-5.

27. Beauchet O, Dubost V, Aminian K, Gonthier R, Kressig RW. Dual-task-related gait changes in the elderly: does the type of cognitive task matter? J Mot Behav 2005;37(4):259-64.

28. Beauchet O, Dubost V, Aminian K, Gonthier R, Kressig RW. Dual-task-related gait changes in transitionally frail older adults: the type of the walking-associated cognitive task matters. Gerontology 2005;51(1):48-52.

29. Van Iersel MB, Ribbers H, Munneke M, Borm GF, Rikkert MGO. The effect of cognitive dual tasks on balance during walking in physically fit elderly people. Arch Phys Med Rehabil 2007;88(2):187-91.

30. Hausdorff JM, Schweiger A, Herman T, YogevSeligmann G, Giladi N. Dual-task decrements in gait: contributing factors among healthy older adults. J Gerontol Ser A Biol Sci Med Sci 2008;63(12):1335-43.

31. Van Iersel MB, Kessels RP, Bloem BR, Verbeek AL, Rikkert MGO. Executive functions are associated with gait and balance in community-living elderly people. J Gerontol Ser A Biol Sci Med Sci 2008;63(12):1344-9.

32. De Brunin ED, Schmidt A. Walking behavior of healthy elderly: attention should be paid. Behav Brain Funct 2010;6:1-8.

33. Hall CD, Echt KV, Wolf SL, Rogers WA. Cognitive and motor mechanisms underlying older adults' ability to divide attention while walking. Phys Ther 2011;91(7):1039-50.

34. Holtzer R, Wang C, Verghese J. The relationship between attention and gait in aging: facts and fallacies. Motor Control 2012;16(1):64-80.

35. Simoni D, Rubbieri G, Baccini M, Rinaldi L, Becheri D, Forconi T, et al. Different motor tasks impact differently on cognitive performance of older persons during dual task tests. Clin Biomech 2013;28(6):692-6.

36. Bidenbaugh SA, Beauchet O, Annweiler C, Allali G, Herrmann F, Kressig RW. Association between dual task-relates decrease in walking speed and real versus imagined Timed Up and Go test performance. Aging Clin Exp Res 2013;25(3):283-9.

37. Tudor-Locke C, Barreira TV, Brouillette RM, Foil HC, Keller JN. Preliminary comparison of clinical and free-living measures of stepping cadence in older adults. J Phys Act Health 2013;10(8):1175-80.
38. Ullmann G, Williams HG. The relationships among gait and mobility under single and dual task conditions in community-dwelling older adults. Aging Clin Exp Res 2011;23(5-6):400-5.

39. Kressig RW, Allali G, Beauchet O. Long-term practice of Jaques-Dalcroze eurhythmics prevents age-related increase of gait variability under a dual task. J Am Geriatr Soc 2005:53(4):728-9.

40. Toulotte C, Thevenon A, Watelain E, Fabre C. Identification of healthy elderly fallers and non-fallers by gait analysis under dual-task conditions. Clin Rehabil 2006;20(3):269-76.

41. Hollman JH, Youdas JW, Lanzino DJ. Gender differences in dual task gait performance in older adults. Am J Mens Health 2011;5(1):11-7.

42. Hollman JH, Youdas JW, Lanzino DJ. Gender differences in dual task gait performance in older adults. Am J Mens Health 2011;5(1):11-7.

43. Donogue OA, Cronin H, Savva GM, O’Regan C, Kenny RA. Effects of fear of falling and activity restriction on normal and dual task walking in community dwelling older adults. Gait Posture 2013;38(1):120-4.

44. Muhaidat J, Kerr A, Evans JJ, Skelton DA. Exploring gait-related dual task test in community-dwelling fallers and non-faller: a pilot study. Physiother Theory Pract 2013;29(5):351-70.

45. Plummer-D'mato P, Altmann LJP, Reilly K. Dual-task effects of spontaneous speech and executive function on gait in aging: exaggerated effects in slow walkers. Gait Posture 2011;33(2):233-7.

46. Al-Yahya, Dawes H, Smith L, Dennis A, Howells $\mathrm{K}$, Cockburn J. Cognitive motor interference while walking: a systematic review and meta-analysis. Neurosci Biobehav Rev 2011;35(3):715-28.

47. Van Loo MA, Moseley AM, Bosman JM, Hassett L. Inter-rater reliability and concurrent validity of walking speed measurement after traumatic brain injury. Clin Rehabil 2003;17:775-9.

48. Wall JC, Scarbrough C. Use of a multimemory stopwatch to measure the temporal gait parameters. J Orthop Sports Phys Ther 1997;25(4):277-81.

49. Bilney B, Morris M, Webster K. Concurrent related valitidy of the GAITRite ${ }^{\circledR}$ walkway system for qualification of the spatial and temporal parameters of gait. Gait Posture 2003;17(1):68-74.

50. Besser MP, Kmieczak K, Schwartz L, Snyderman M, Wasko J, Selby-Silverstein L. Representation of temporal spatial gait parameters using means in adults without impairment. Gait Posture 1999;9(2):113. 